\title{
COOPERATIVE AND APPLICATION-ORIENTED LEARNING IN ENGINEERING DESIGN - SYSTEMS DESIGN METHODOLOGY EDUCATED ON SOLUTIONS FOR MOUSETRAP-POWERED VEHICLES
}

\author{
Jerome KASPAR and Michael VIELHABER \\ Institute of Engineering Design, Saarland University
}

\begin{abstract}
According to recent studies, scientists and trend researchers are both quite confident that changes within the next two decades will be much more extensive and pioneering than the changes during the past 20-25 years. Having all the emerging technological trends and visions in mind (e.g., artificial intelligence or the IoT platform), the complexity will achieve an entirely new dimension within our knowledge-based society. In order to be successful in future, engineers or actually young aspirants need to change their learning behaviour to overcome these new issues in complexity management. Hence, cooperative and application-oriented learning including an exchange of knowledge and experience is increasingly gaining in importance, especially in view of the abstract and holistic thinking within the field of systems engineering.

Dealing with this future complexity and difficulty, this contribution presents an innovative manner of transferring knowledge in a considerably practice-oriented way within the largely theory-based subject of "systems design methodology" for first semester bachelor students. In doing so, the pursued systematic product development procedure deals with the prospectively more important aspects of an integrated view on product design, material and technology selection as well as soft skills issues such as showing individual initiative, innovative spirit and time management, apart from social competences (e.g., appropriate teamwork skills as well as a strong ability to communicate and critical faculty).
\end{abstract}

Keywords: Design education, engineering design, systems design methodology, theory and practice

\section{INTRODUCTION AND CONTEXT}

Today, product design is a complex and multifaceted discipline necessitating a wide range of competencies and skills. Thus, the industrial use of methodological approaches to solve abstract issues or technical tasks systematically at an early development stage has been increasing in recent years, notably considering interactions of software and systems engineering [1]. Model-based systems engineering (MBSE), for example, constitutes one specific and widespread option regarding a formalised application of a functional description and design of system architectures, i.e. a supported modelling of requirements, design, analysis, verification and validation activities as a primary means of an interdisciplinary, creative and consistent information exchange and data management, particularly for the conceptual design phase [2]. In this context, advanced (graphical) modelling languages such as SysML are being used to decompose and specify the system process regarding structure, behaviour, requirements, and parametric. More recently, and to extend the focus on respective simulation aspects steadily growing in importance, modelling and simulation-based systems engineering (M\&SBSE) efforts are state of the art to further increase productivity [3].

Against this background of a needed reconsideration of today's methods, processes, IT solutions, and organisational structures, future innovative product developments require an early sensitisation and education of its engineering personnel. Accordingly, to find an easy entrance to this thematic field, an innovative manner of transferring knowledge within the subject of "systems design methodology" is targeted in a practice-oriented way for first semester bachelor students based on the fundamental principles of Pahl and Beitz [4], which is now being introduced. Thus, the educational framework is 
presented first (section 2), before going ahead with the actually aimed cooperative and applicationoriented learning concept in section 3 as well as its ensuing evaluation.

\section{EDUCATIONAL FRAMEWORK AND REQUIREMENTS}

In order to provide the necessary framework for an enhanced learning concept, the educational framework as well as the required student demands within their project-oriented lecture is initially emphasised by giving a short project description and highlighting the desired objectives.

\subsection{Subject and Goal of Lecture}

The subject of "system design methodology (1)" is the first course of a two-stage series within the graduate bachelor's / master's structure of the newly introduced systems engineering study programme. Within the (first semester) undergraduate level course, the actual content is divided into an intended theoretical (lecture) and practical (tutorial) part. The freshmen acquire basic knowledge and skills in the field of systems engineering, product development, and technical design. The content includes the overview of the general product lifecycle and its historically established product development processes, apart from more detailed insights within comprehensive and domain-specific development methodologies and modelling approaches, but also contains a slight introduction into the technical drafting, virtual (computer-aided) development, and project management in the second half of the semester term. This knowledge transfer can be extremely tedious, but indispensably needs to be educated and learned from scratch to systematically develop future products most efficiently. For this reason, the fundamental knowledge sharing in theory and the regular exercises towards its further deepening, our education research includes an innovative manner of transferring this extensive knowledge additionally in a more practice-oriented and self-reliant way gathering their own experiences by implementing a parallel and term-accompanying practical project within the comprehensive lecture programme.

The project-intensive subject discusses all essential phases from the initial product planning (simplified market, competition, technology and product analysis), through the task clarification as well as conceptual, embodiment and detail design (manually created fundamental technical drawings leading to 3D-CAD-models), up to a prototypical realisation succeeding in a public contest. In this way, diverse physical basics such as motion and energy aspects, mechanical principles, as well as surface and fluid friction issues are put into context of modifications in design, function or technology, which serves as an inevitable engineering basis. The students learn how to work on a future typical workflow of temporary project-based activities coupled with its challenges regarding an agile and tight project management (on-time completion, unassisted but efficient concept development and responsibility, and agreement on joint solutions). Originating from the selection of a low budget but no less complex and visual project task of a prototypical conception of a small, funky mousetrap-powered vehicle, an ultimate weak-analysis - carried out on the self-constructed engineering solution - enables a further educational process due to well-motivated re-design thoughts driven by ambition.

\subsection{Project Description}

Based on this partly innovative procedure of knowledge mediation, and against the background of taking up media-driven discussions all about the actual climate change policy, the proposed project task deals with a simplistically playful way to think methodologically about alternative drive systems to steadily replace conventional vehicles for air, land and water endangering human health and polluting the environment. To address this specific aim in the context of prematurely making the idea of green mobility a palpable and, at the same time, enthusing vision, initially mousetrap-powered cars followed by boats, gliders and - at present - amphibians are built as scale models by all student teams as a more practical preparation and dedicated precondition for admittance to the exam.

For this purpose, the applicable technical regulations specify the fundamental framework conditions already at the baseline including information, on allowed modifications of the designated mousetrap itself (e.g., max. four holes exclusively for attaching the mousetrap to the construction frame and/or slight changes in bending the hammer in terms of extending the lever arm, but no additional turns of the spiral spring with maximum 180 degree angular deflection), the maximum dimensions, weight and costs of the vehicle design, or the general prohibition of further potential, kinetic and/or ulterior energy at the start, except for the energy generated and stored by the mousetrap. Based on this design task particularised in the "customer-oriented" specification sheet, students in a group of two or three 
progressively approach a feasible overall solution being optimally developed and selected according to the product development process imparted in the course, and finally being prototypically put into practice for the prescribed competitive terrain. Considering a more comprehensive presentation of the upcoming application-oriented learning concept, the specific task of designing an amphibian vehicle (powered by two mousetraps) is primarily demonstrated in an exemplary way as a mixture of consecutive shore leave ( 3 metre) and waterway (1 metre).

\section{COOPERATIVE AND APPLICATION-ORIENTED LEARNING CONCEPT}

Having clarified the subject and goal of the lecture along with a further description of the included project, subsequently the cooperative and application-oriented learning concept is presented.

\subsection{Education Strategy}

The educational procedure fundamentally pursues the product development process according to Pahl and Beitz [4] added by the more holistic view regarding an integrated definition of product design, material and production engineering (IPPE) according to [5], see Figure 1.

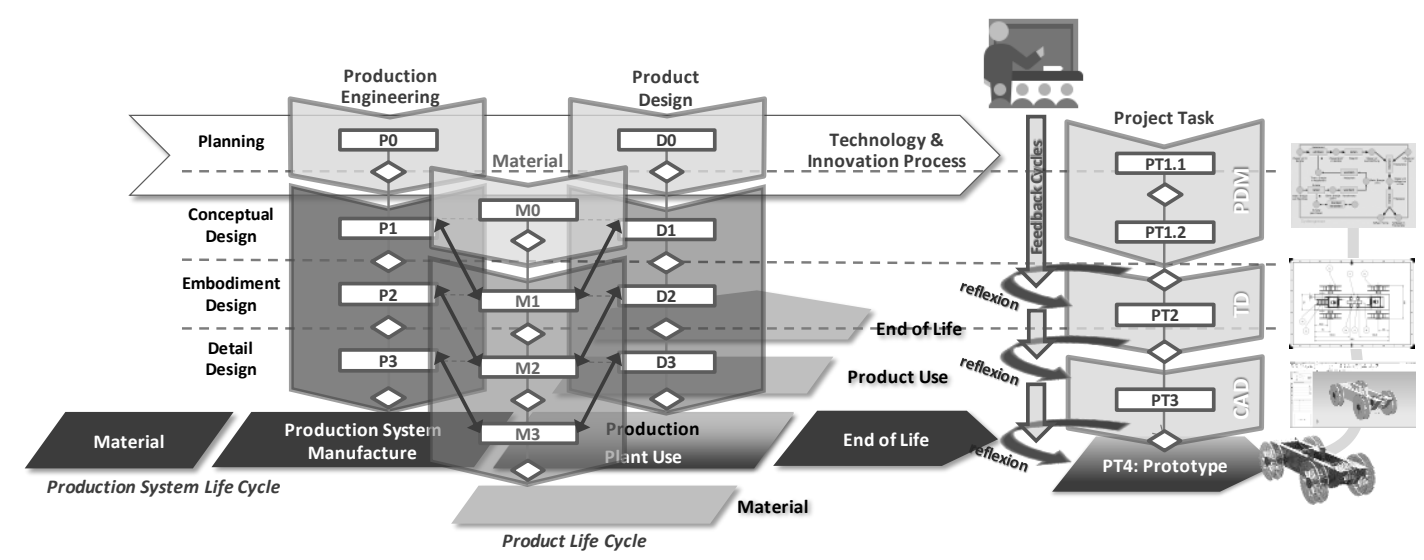

Figure 1. Educational framework of the application-oriented learning concept, according to [5]

The individual project tasks derived from the basic IPPE framework [5] successively guides the students systematically from an idea or vision to the prototypical built (PT4), whereas each individual completion of the product development methodology (PT1.1 \& PT1.2), technical drawing (PT2) and computer-aided design (PT3) stage passes an evaluated feedback cycle with the teaching staff needed to achieve a minimum level of success and reflect upon a further optimised product system.

In doing so, especially for the initial stage of planning and conceptual design, the visionary concept is additionally to be highlighted in a 10-minute presentation plus discussion in front of a professional (academic) jury and elected students from the last-year project in the sense of a design competition, i.e. explaining how the individual design works, justifying the choice of working principles chosen over another, pointing out particular benefits, and finally identifying selected weaknesses with suggestions for improvement. As a result, the judged students' comments provide decisive information about a further proceeding and optimisation due to their past experiences from the same situation last year whilst the elected students themselves in return gain rewarding expertise in briefly assessing products and learn to give useful feedback. Apart from that, and as for all other project parts, a written submission of the required data set is needed. These re-evaluated documents (given the fact of its prequalification status of acceptance on the exam) are respectively discussed further on in brief personal advisory discussions with respect to proposed enhancements and hints in the technical design (PT2) and CAD-modelling (PT3) just before the real finalisation of the product system commences. Consequently, a cooperative and learning-oriented ambience is created for the admission restriction.

Completed by the self-reliant assembly of a prototype (PT4) and its emerging challenges physically being evident while having the functional tests in reality differing from the ideal conditions or hidden pitfalls in the simulation environment, ultimately the educated theory in the lecture being deepened in the collective tutorial sessions could be further broadened by the independent and practical application project in small groups and its consultations and exchange there or with the teaching staff (including the supportive last-year student advisory board). 
As a result, especially integrated considerations on the decisive interaction of materials and its processing additionally with respect to an appropriate and feasible design (i.e., prescribed restrictions and needed modifications) are pursued right from the conceptual beginning to the technical modelling and shaping of the built prototype in the end. This education strategy is now being discussed in more detail for the mousetrap-powered vehicle project, particularly the amphibian model.

\subsection{Applied Learning Concept with Student Outputs - Mousetrap-powered Vehicle}

Being based on the project task and beginning with the project planning phase (PT1.1), students initially have to set a time and cost framework with a general target and its temporal, financial, personal and other limitations ensuring that deviations and problems can be efficiently detected and addressed already at an early stage. Thus, the preparation of a Gantt chart displaying bar graphs for the individual stages of the project realisation (e.g., partly subdivided into vehicle functions) on a horizontal time scale is aimed for the allocation of operations to resources and their starting time, duration and (critical) dependencies being enhanced with a colour-coding and an auxiliary text display for relevant data. At the same time, a timely recognition and clear addressing of market (type and volume), competition and technology trends is focused to systematically search and select or basically adapt future-oriented product innovations being premised on related solutions.

The methodological procedure gets started with substantial considerations on the conceptual design (PT1.2). Here, setting up the mandatory requirements list is initiated first being classified in product design (D0), material (M0) and production engineering ( $\mathrm{P} 0)$ demands. It points out how, amongst others, the influence of the material selection depends on environmental aspects (e.g., water resistance and floating). With the aid of a consistency matrix, negative or conflicting key requirements could be determined and exploited due to their significant potentials for innovation (e.g., lightweight design (efficiency) and long wheelbase (driving behaviour) vs. stiffness (trap forces) $=$ sandwich construction). Next, and starting from the gathering of input (e.g., potential energy) and output (e.g., kinetic energy and buoyancy force) as well as loss (e.g., friction) and disturbance (e.g., weather conditions) variables by means of the cybernetic black box representation, a successive splitting of the required overall function (hierarchically functional structure) and their sub-functional link (conversion of energy, material, signals [4]) to a meaningful overall functional model is mainly traced to get a deeper penetration of the conceptual formulation. Thus, either the release of the mousetraps in a row or at the same time leads to a steady transformation of the energy transmitting the torque to an efficient movement as amphibian first on shore and second in water. Consequently, the goal of thinking in functions is the detachment from the objective (concrete) and premature determinations of a particular solution principle. Having done these fundamentals on functional level, the selection of a most appropriate working combination requires the creation of individual working principles. Using the morphological charts method constitutes one possible option to list all mutually suitable principles for their underlying functions by applying different solution identification methods such as solution catalogues (search method), Brainstorming, Six Thinking Hats or Lateral Thinking (creative methods) as well as TRIZ or USIT (systematic methods). Hence, even less obvious solution principles are considered without prematurely loosing concept alternatives right at the beginning. For example, the activation of the second mousetrap initiated through an innovative construction using the buoyancy of an object attached to the lever arm (option 2, Table 3) right when plunging into the water just like the intended merger of an open wheel with segmented paddles performing as a combined drive system (listed option 1). This allows a clear selection of objectively best working combinations pursuant to a chosen evaluation system (e.g., pairwise comparison, ranking or two-dimensionally technicaleconomic evaluation respectively with a different weighting, scale, and choice of criteria) regarding previously checked individual compatibilities of working principles across the functional structure. A viable mix must be made between a highly weighted performance and lightweight material costs without losing to much efficiency through friction losses in the driving system or mechanical force transmission due to resultant economic restrictions. By means of applying a value profile for the choice of eligible concept alternatives, detected weaknesses could be resolved by last adjustments within the working principles. As a result, originating from a comprehensive choice of validated working combinations, the best possible principle solution is selected for a further elaboration provisionally sketched as a schematic illustration.

Project task 2 now contains the embodiment design concerning the technical drawing. The designed product must be completely documented hand-drawn with all necessary parts and assembly drawings, 
a sectional view of the assembly in longitudinal direction as well as parts lists or bill of materials and other documents (e.g., short specification of purchased parts) for a smooth production process without problems. Here, particular attention is given to a consistent and exhaustive technical dimensioning in the standardised arrangement of the part projection views including purposively dimensional, geometrical and positional tolerances along with surface details suitable for production. To assemble the production in the end, the parts list referred to the positions on the assembly drawing is added by the information about the material type and its costs when faced with a subsequent cost calculation listing material and production costs as well as prime costs (for development, construction, administration, sale, and marketing) to determine the sales price with an imputed profit.

In project task 3 the initial two-dimensional product is transformed into a three-dimensional CAD model, as depicted in Table 1. Apart from the generation of the visually more informative 3D-CAD data sets of the individual parts and assembly, the simplistically physical modelling of the various degrees of motion needs to be simulated corresponding to their real operating principle. First predictions regarding its performance could be indicated in addition to the digitally derived technical drawings directly facilitating a computer-operated machining.

Table 1. Different but successful concepts of mousetrap-powered vehicles

\begin{tabular}{|l|l|l|l|}
\hline Mousetrap Car & Mousetrap Boat & Mousetrap Glider & Mousetrap Amphibian \\
\hline & &
\end{tabular}

Finally, and based on all these preliminary stages, the construction of a prototype is striven in project task 4 . Here, the self-reliant and physical assembly and perceptible testing of the internally developed product system again highlights areas with further potential for optimisation resulting in last design modifications, especially for the prestigious distance and speed contest against the other student teams.

\section{EMERGED CHALLENGES, PROJECT EVALUATION AND CONCLUSION}

Having experienced four semesters of conducting such a kind of an almost unchanged cooperative and application-oriented lecture, many general challenges become visible (e.g., getting the most out of a smart knowledge transfer or even understanding student difficulties from their point of view).

Starting with the student challenges with respect to the educated framework of an integrated definition of product design, material and production engineering, the complexity of interrelations of a manufacturable product system is striking at the very first moment when being faced with all the prescribed technological, economical and/or ecological restrictions. Here, and apart from the major difficulties with an intuitively concrete selection of expedient tolerances within the standardised technical drawing, especially the initial solution-neutral conception setting up interrelated functions causes several uncertainties due to their unfamiliar abstract character originating from the continuous desire for more conceivable principles. By systematically introducing practical application examples prior to the novel gain of own (also haptic) experiences during the actual project task supported by iteratively reflected feedback cycles (particularly including the last-year students advisory board). The students relatively easy come up with the nowadays still absolutely indispensable feeling for an operationally function-based thinking, specific mechanical dimensions, as well as geometric and surface tolerances per common combination. Perceiving this comprehensive benefit within the modern process of engineering education, the repeatedly seen management of the whole project as 
"overexerting from the perspective of a freshman with hardly any knowhow of product design" in the beginning gets mostly changed at the end of the semester in similar statements as listed below, which is furtherly being favoured in the student evaluations depicted in Figure 2 (left).

"[...] To conclude, a positive thing about 'systems design methodology' is that there isn't just the engineering part, you also learn a lot about materials and processing, time management as well as business economics (budgeting) not only in theory but also in practice. I myself am a big fan of this 'learning-by-doing' concept and learned way more because I was forced to find tailored solutions for the various difficulties which emerged during the development process (e.g., importance of a proper assembly design) while understanding the physics behind this in reality."

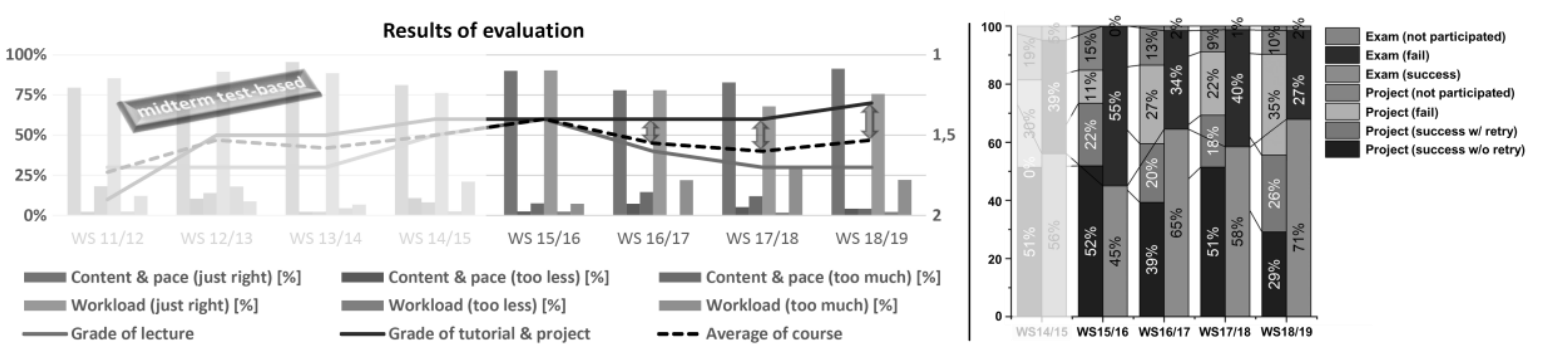

Figure 2. Development of evaluation and exam (incl. midterm) results

Picking up the positive student evaluations despite its considerable extra workload (exception: "surprising effect" of first year) since the replacement of the regular midterm tests (steadily increasing positive delta between grade of tutorial \& project compared to course average) by a project-based education environment, a critical reflection of the learning outcomes can initially be provided on the basis of the historical exam results. Although Figure 2 (right) does not clearly highlight the assumed impact of a tougher project part evaluation on the success rate of the final exam (winter semester 2016/17 vs. 2018/19), at least a slight improvement of the average grade of the exam (overall optimum of $\varnothing 3,0)$ at a slightly decreased failure rate of less than $30 \%$ has been achieved corresponding to the successively optimised education strategy. Nonetheless, an absolute statement for the holistic engineering learning output cannot be given on that database due to the substantial differences of the annually changed intellectual (educational background and attitude) variations of the students group, modified exam questions, and ultimately mainly focused examination on the subject matter of system design methodology and technical drawing without a further soft skill or project management audit.

Against this background of a consistently positive response and outcome of the introduced learning concept and education strategy, the next semester project will induce more flexibility in the own choice of a fully sustainable power unit to furtherly stimulate the process of visionary thinking. Future activities will take place to higher academic courses, for example, the systematic development of an "innovative household gadget in a partly additively manufactured multi-material design" where the task is to functionally optimise one existing or to skilfully combine several articles. Thus, applicationoriented issues get broader significance in the comprehensive engineering education.

\section{REFERENCES}

[1] Boehm B. Some Future Trends and Implications for Systems and Software Engineering Processes. Systems Engineering, 2006, 9(1), 1-19.

[2] Walden D.D., Roedler G.J., Forsberg K.J., Hamelin R.D. and Shortell T.M. INCOSE Systems Engineering Handbook: A Guide for System Life Cycle Processes and Activities, 4th edition, 2015 (John Wiley \& Sons, Hoboken, NJ).

[3] Gianni D., D'Ambrogio A. and Tolk A. Modelling and Simulation-Based Systems Engineering Handbook, 1st edition, 2014 (CRC Press / Taylor \& Francis Group, Boca Raton, FL).

[4] Pahl G. and Beitz W. Engineering Design: A Systematic Approach, 2nd edition, 1996 (SpringerVerlag, London).

[5] Stoffels P., Bähre D., Frey G. and Vielhaber M. Energy efficiency engineering - towards an integrated method framework for energy-oriented product and production development. In: Oral A.Y. et al., editors. 2nd International Congress on Energy Efficiency and Energy Related Materials (ENEFM2014). Cham (CH): Springer, 2015, pp. 291-297. 